\title{
Compressive strengths of PEG gels with glycerol and bioglass particles
}

\author{
Ariel Golshan \\ Department of Engineering, Hofstra University, Hempstead, NY 11549, USA \\ Jenesis A. Curtis \\ Department of Biology, Hofstra University, Hempstead, NY 11549, USA \\ Vasilios Lianos \\ Department of Biology, Hofstra University, Hempstead, NY 11549, USA \\ Sina Y. Rabbany \\ Bioengineering Program, Department of Engineering, Hofstra University, Hempstead, NY 11549, USA \\ Roche C. de Guzman ${ }^{\text {a) }}$ \\ Bioengineering Program, Department of Engineering, Hofstra University, Hempstead, NY 11549, USA \\ a) Address all correspondence to this author. e-mail: roche.c.deguzman@hofstra.edu
}

\begin{abstract}
Poly(ethylene glycol) (PEG)-based materials can potentially be used as biomechanical matrices for regenerative medicine implants including the replacement of intervertebral (IV) discs.

Glycerol and other plasticizers (low-MW PEG, propylene glycol, and sorbitol) were added to the bulk PEG matrix, gelled using chemical and photochemical methods at different temperature and pressure settings, and compression properties acquired and analyzed. Incorporation of surface bioactive glass particles shortened the blood clotting time, while alginate and laponite additives improved the gel's mechanical properties to $645 \mathrm{kPa}$ compressive modulus, $12 \%$ yield strain, and $79 \mathrm{kPa}$ yield strength. This IV disc-modeled system endured the cyclic loading and unloading test indicative of an elastic response; but required improvement of its biomechanical tolerance.
\end{abstract}

Keywords: biomedical; stress/strain relationship; strength 


\section{INTRODUCTION}

Poly(ethylene glycol) (PEG) is a commonly-used biomaterial due to its proven safety record, nontoxicity, and biocompatibility. ${ }^{1-5}$ However, as tissue engineering materials specifically for intervertebral (IV) disc applications, PEG hydrogels alone are not sufficient due to their inherent fragility.$^{6-9}$ Plasticizers are low-molecular weight (MW) compounds that increase the flexibility of polymers by filling-up the network spaces, allowing increased polymer mobility. ${ }^{10-12}$ In this study, to improve the compressive strengths and other important mechanical properties of PEG hydrogels, U. S. Food and Drug Administration (FDA)'s generally recognized as safe (GRAS) substances: glycerol (glycerin), propylene glycol, and sorbitol, and relatively small-MW uncrosslinked PEG were utilized as plasticizers (Table I). The crosslinking process of the bulk PEG matrix was conducted using PEG diacrylate (PEGDA) chain intermediates, and the effect of chemical versus photochemical PEGDA chain elongation leading to gelation, was also investigated at various temperature and pressure conditions, as they (type of crosslinkers, temperature, and pressure) affect the physical properties of gels. ${ }^{13-17}$

Alginate biomaterials, the naturally-derived polysaccharides that gel via chain interactions linked by multivalent cations (such as $\mathrm{Ca}^{2+}$ ), have been utilized for IV-disc regeneration research. ${ }^{8,18,19}$ Laponite is a mixture of inorganic minerals with two-dimensional (2D) diskshaped architecture for the improvement of bulk mechanical properties. ${ }^{20}$ Together, these additives (alginate and laponite) were incorporated in the PEG gel assembly to potentially sustain the loads experienced by native lumbar IV discs. ${ }^{8,18,21}$ Finally, bioactive glass or bioglass ${ }^{22}$ particles, the engineered slowly-degrading materials known to bind to bones without inducing a foreign-body capsule reaction, were synthesized in the lab then layered onto the surfaces of the cylindrical gel and evaluated for blood clotting to imply host tissue integration.

\section{EXPERIMENTAL}




\section{A. Materials}

The following chemicals were purchased from Sigma-Aldrich (St. Louis, MO): poly(ethylene glycol) diacrylate (PEGDA; MW = $700 \mathrm{~g} / \mathrm{mol}$ ), glycerol (GLY; MW = $92 \mathrm{~g} / \mathrm{mol}$ ), poly(ethylene glycol) (PEG; MW = 8000 g/mol; n 181), propylene glycol (PRO; MW = 76 g/mol), sorbitol (SOR; MW = $182 \mathrm{~g} / \mathrm{mol}$ ), ammonium persulfate (APS), tetraethylethylenediamine (TEMED), methanol $(\mathrm{MeOH})$, phosphate-buffered saline $(\mathrm{PBS})$, silica $\left(\mathrm{SiO}_{2}\right)$, sodium oxide $\left(\mathrm{Na}_{2} \mathrm{O}\right)$, calcium oxide $(\mathrm{CaO})$, phosphorus pentoxide $\left(\mathrm{P}_{2} \mathrm{O}_{5}\right)$, calcium chloride $\left(\mathrm{CaCl}_{2}\right)$, and sodium alginate (ALG; product \# W201502). Irgacure ${ }^{\circledR} 2959$ (I2959) was obtained from BASF (Vandalia, IL) and Laponite ${ }^{\circledR} \mathrm{RD}$ (LAP; synthetic layered silicate made from $\mathrm{SiO}_{2}, \mathrm{MgO}, \mathrm{Li}_{2} \mathrm{O}$, and $\mathrm{Na}_{2} \mathrm{O}$ ) from BYK Additives (Gonzales, TX). Deionized water was used as the solvent for preparing solutions unless stated otherwise.

\section{B. Gel synthesis using photochemical-crosslinking with varying plasticizers}

Cylindrical gels were prepared into wells of 24-well poly(styrene) plates at final concentrations of: 20\% (V/V) PEGDA, 10 mg/mL I2959 (diluted from 100 mg/mL I2959 in $\mathrm{MeOH}$ ), and varying amounts of plasticizers (GLY, PEG, SOR, and PRO) up to their saturation limits. The saturation limits of respective plasticizers in gels were determined by adding increasing amounts of plasticizers (10\% to $75 \%$ ) until the gel could no longer hold the plasticizer

(phase separation observed). The maximum amounts of gel-incorporated plasticizers were found to be: $\mathrm{GLY}$ at $40 \%(\mathrm{~V} / \mathrm{V})=0.4 \mathrm{~mL} / \mathrm{mL}=504 \mathrm{mg} / \mathrm{mL}, \mathrm{PEG}$ at $30 \%(\mathrm{~m} / \mathrm{V})=300 \mathrm{mg} / \mathrm{mL}, \mathrm{PRO}$ $70 \%(\mathrm{~m} / \mathrm{V})=700 \mathrm{mg} / \mathrm{mL}$, and SOR at $75 \%(\mathrm{~m} / \mathrm{V})=750 \mathrm{mg} / \mathrm{mL}$ (Table I). These values were then set to be the $100 \%$ relative saturation levels. PEG, SOR, and PRO were prepared at higher concentrations prior to dilutions. After mixing the components into uniform solutions, samples were photo-crosslinked by exposure to a 36-W, 254-nm UV light for 20 minutes at ambient temperature $\left(21^{\circ} \mathrm{C}\right) .^{23}$ 


\section{Gel compression analysis}

Strength and viscoelastic properties of gels were characterized through unconfined compression until fracture. Freshly-made gels were biopsy-punched to obtain cylindrical specimens with diameter $(\mathrm{d})=11 \mathrm{~mm}$, loaded onto a platen, then compressed with a parallel blunt probe at a speed of $0.1 \mathrm{~mm} / \mathrm{s}$ using the Instron 3345 mechanical tester (Norwood, MA) with a 100-N load cell. Settings were configured and data (relative displacement versus force) were acquired with the Bluehill 3 software (Instron). Data were processed in Excel (Microsoft, Redmond, WA) and MATLAB (Mathworks, Natick, MA) to obtain true stress and strain results. ${ }^{24}$ Compression properties: elastic modulus at compression $\left(\mathrm{E}_{\mathrm{c}}\right)$, and yield and ultimate strains and stresses were determined.

\section{Gel synthesis using chemical crosslinking at different temperatures and pressures}

PEG-based gels were fabricated via chemical crosslinking by homogenously mixing the following: 20\% (V/V) PEGDA, 30\% (V/V) GLY plasticizer, $1 \%$ (V/V) TEMED, and $1 \mathrm{mg} / \mathrm{mL}$ APS into the chamber of a heated die set (Across International, Livingston, NJ). A $10 \mathrm{mg} / \mathrm{mL}$ APS intermediate solution was prepared and used prior to the gelation reaction. Gels $(\mathrm{d}=25 \mathrm{~mm}$ and $\mathrm{h}=5 \mathrm{~mm}$ ) were allowed to set at different temperatures: $21,37,59$, and $80{ }^{\circ} \mathrm{C}$ using a temperature controller for 15 minutes, equilibrated back to room temperature (RT), biopsypunched at $\mathrm{d}=8 \mathrm{~mm}$, and unconfined compression testing conducted as described above. Gels without the GLY plasticizer were utilized as controls. Representative PEG-GLY gels were also fabricated under $90 \mathrm{MPa}$ of gauge pressure (compared to gels made in atmospheric pressure) for 15 minutes at $21^{\circ} \mathrm{C}$ within an MP15 desktop pellet press (Across International).

\section{E. Glycerol release assay}

The stability of GLY in PEG-GLY gels were tested by spectrophotometry. Hydrogels (20\% PEGDA and 30\% GLY) were made similar to the abovementioned protocol using chemical 
crosslinkers. 8-mm diameter samples were obtained using a biopsy puncher and these gels were placed into wells of a 48 -well microplate. $300 \mu \mathrm{L}$ of PBS was added into wells, incubated at RT, and at timepoints: $0,4,12,24,74$, and 168 hours (or at $0,0.2,0.5,1,3.1$, and 7 days), liquid samples were collected and replaced by fresh PBS. Spectral scans of these specimens were conducted using the NanoDrop spectrophotometer (Thermo Fisher Scientific, Waltham, MA) at 190 to $200 \mathrm{~nm}$ with PBS as a blank. Chemicals that can potentially leach out of the PEG-GLY gels (PEGDA, GLY, TEMED, and APS) were tested as well. The absorbance values were computed as the difference between samples and the blank PBS at $193 \mathrm{~nm}$. GLY concentration was determined using the linear trendline of the glycerol standard curve. Mass release fraction was computed by dividing the released glycerol mass with the initial mass. A scatterplot of cumulative mass release versus time was then generated and a saturation curve fitted using leastsquares regression in MATLAB with the equation:

$$
\mathrm{R}(t)=\mathrm{R}_{\max }\left(\frac{\mathrm{t}}{\mathrm{t}_{\mathrm{h}}+\mathrm{t}}\right)
$$

where $\mathrm{R}=$ cumulative mass release fraction (function of $\mathrm{t}$ ), $\mathrm{t}=$ time, $\mathrm{R}_{\max }=100 \%$ (maximum release), $\mathrm{t}_{\mathrm{h}}=$ saturation equation constant $=$ time to release half the load.

\section{F. Bioactive glass synthesis and incorporation into gels}

Bioactive glasses (BGHU1 and control Bioglass 45S5 (BG45S5)) were fabricated based on the modified protocol of Greenspan and Hench. ${ }^{25}$ Briefly, $50 \mathrm{~g}$ total of chemical powders: $\mathrm{SiO}_{2}$, $\mathrm{Na}_{2} \mathrm{O}, \mathrm{CaO}$, and $\mathrm{P}_{2} \mathrm{O}_{5}$ were homogenously mixed into a ceramic crucible (Engineered Ceramics, Gilberts, IL) and melted in an electric kiln at $1288^{\circ} \mathrm{C}$ for 4 hours. The theoretical calcium to phosphorus mole ratios $\left(\mathrm{n}_{\mathrm{Ca}} / \mathrm{n}_{\mathrm{P}}\right)$ of BGHU1 and BG45S5 were 4 and 5 , respectively. Using metal tongs, the liquified glass was poured into new crucibles in another kiln set at $460{ }^{\circ} \mathrm{C}$, incubated for 8 hours (annealing), and allowed to cool to room temperature for 24 hours. The synthesized glasses were removed from the crucible, and ground into fine particles with a mortar and pestle. 
PEG (20\% PEGDA)-GLY (30\%)-bioglass gels were made with 5\% (m/V) bioactive glass particles using chemical crosslinkers. After the gelation process, additional 5\% bioglass was added on the gel surface.

\section{G. Blood coagulation test}

To investigate the potential host response effect of bioactive glass additives on gels, a clotting assay was performed. PEG-GLY-bioglass gels (PEG-GLY-BGHU1 and PEG-GLY-BG45S5) were made at $1.5 \mathrm{~mL}$ each into wells of a 12-well microplate. Control groups: no gels (surface of the poly(styrene) plate), PEG only, and PEG-GLY gels were also included. Samples were quickly rinsed with PBS to remove excess and unreacted reagents. Cat bloods in EDTA tubes collected from animal shelters with an approved Animal Care and Use Committee (ACUC) protocol from Dr. John Foy (Bobbi and the Strays, Freeport, NY) at $100 \mu \mathrm{L}$ were mixed with $1 \mathrm{M}$ $\mathrm{CaCl}_{2}$, then immediately added onto the test gel substrates. Blood coagulation time was recorded and normalized over the surface area of blood contact.

\section{H. Addition of alginate and laponite in hydrogels}

To potentially improve the strength properties of PEG-GLY-BGHU1 constructs, alginate (ALG) and laponite (LAP) biomaterials were added. Specifically, PEG-GLY-BGHU1-ALGLAP gels were made by mixing 20\% PEGDA, 30\% GLY, 5\% BGHU1, 2.5\% (m/V) ALG and $2.5 \%(\mathrm{~m} / \mathrm{V})$ LAP, then chemically crosslinked by $1 \%$ TEMED and $1 \mathrm{mg} / \mathrm{mL}$ APS. After curing for 15 minutes, gels were soaked in $1 \mathrm{M} \mathrm{CaCl}_{2}$ solution overnight to polymerize the alginate components. Compression until failure test was performed and properties obtained.

\section{Cyclic compression loading}

Bigger PEG-GLY-BGHU1-ALG-LAP cylindrical gels were fabricated (at $\mathrm{d}=50 \mathrm{~mm}$ and $\mathrm{h}=$ $10 \mathrm{~mm}$ ) to model a human intervertebral (IV) disc between vertebrae lumbar 3 and 4 (L3 and L4). 
L3 and L4 vertebral bodies were simplified, modeled as cylinders that slightly taper in the middle, and three-dimensional (3D)-printed using a green poly(lactic acid) (PLA) filament (MakerBot, Brooklyn, NY). The hydrogel "IV disc" was positioned between PLA "vertebrae" and placed on the platen of Instron 3345. The Bluehill 3 software was configured for cyclic loading at maximum compressive strain of $4 \%$ (at $0.1 \mathrm{~mm} / \mathrm{s}$ ) for 100 cycles of compression and relaxation. Stress-strain curves were plotted. Dissipated energy density was calculated as the area of the hysteresis loop using MATLAB. A power equation was fitted using the formula:

$$
\mathrm{E}(n)=\alpha \mathrm{n}^{\beta}
$$

where $\mathrm{E}=$ dissipated energy density (function of $\mathrm{n}$ ), $\mathrm{n}=$ cycle number, and $\alpha$ and $\beta=$ constants.

\section{J. Statistical analyses and softwares}

Experimental samples were performed in triplicates $(n=3)$. Values were reported as mean \pm 1 standard deviation. Student's t-test and one-way analysis of variance (ANOVA) with multiple comparison (Tukey's) tests were performed in MATLAB at 5\% probability (p) of type I error. Chemical structures of plasticizers were drawn using ChemSketch (Advanced Chemistry Development, Toronto, ON, Canada).

\section{RESULTS AND DISCUSSION}

\section{A. Compression properties of bulk PEG gels with GLY, PEG, PRO, and SOR}

Relative displacements and compressive loads (forces) were converted to true compressive strain and stress values, respectively, and plotted to obtain the elastic modulus at compression or compressive modulus $\left(\mathrm{E}_{\mathrm{c}}\right)$, yield, and ultimate strain and stress properties [Fig. 1].

The shapes of the stress-strain curves of PEG gels (using 20\% PEGDA) at varying plasticizer saturation concentrations changed expectedly; steepness of slopes increased then decreased [Fig. 2(a) showing PEG-GLY gels]. Most PEG gels made via photochemical polymerization did not 
fracture during the compression test. Hence, only the $\mathrm{E}_{\mathrm{c}}$, yield stress $\left(\sigma_{\mathrm{c}-\mathrm{y}}\right)$, and yield strain $\left(\varepsilon_{\mathrm{c}-\mathrm{y}}\right)$ data were extracted from the stress-strain scatterplots. Plots of these properties versus plasticizer saturation [Fig. 2(b)-2(d)] revealed some patterns. The $\mathrm{E}_{\mathrm{c}}$ values (slopes of the linear elasticity region) generally increased then decreased from 0 to $100 \%$ saturation [Fig. 2(b)] corresponding to plasticizing and anti-plasticizing effects ${ }^{26}$, respectively. PEG gels with $60 \%$ saturated SOR (or $750 \mathrm{mg} / \mathrm{mL} \times 60 \%=450 \mathrm{mg} / \mathrm{mL}$ SOR $($ Table I) $)$ produced the highest $\mathrm{E}_{\mathrm{c}}$ at $1.5 \mathrm{MPa}$. The staggered conformations of sorbitol ${ }^{27}$ possibly got entangled within the PEG matrix (at $60 \%$ saturation) leading to the highest stability and elasticity among the tested plasticizers. In other studies, sorbitol plasticizer also increased the strength properties of structural proteins films..$^{28,29}$ Glycerol, propylene glycol, and PEG 8000 (PEG with MW = 8000) also exhibited plasticizing effects at low saturation levels: 25\%,14\%, and 50\%, respectively [Fig. 2(b)].

Addition of small amounts of plasticizers decreased the gel flexibility (decrease in $\varepsilon_{\mathrm{c}-\mathrm{y}}$ ) but reverted to original values (of $8 \%$ strain) at plasticizer full saturation levels except for the gel with $100 \%$ saturated SOR that increased the $\varepsilon_{\mathrm{c}-\mathrm{y}}$ to about $13 \%$ [Fig. 2(c)]. Compressive yield stresses erratically fluctuated at different plasticizer quantities [Fig. 2(d)]. Gels with $450 \mathrm{mg} / \mathrm{mL}$ SOR recorded $0.074 \mathrm{MPa}=74 \mathrm{kPa}$ of $\sigma_{\mathrm{c}-\mathrm{y}}$. Interestingly, the PEG-based gels with $75 \%$ saturated GLY (or $0.4 \mathrm{~mL} / \mathrm{mL} \times 75 \%=0.3 \mathrm{~mL} / \mathrm{mL}=30 \% \mathrm{GLY}$ ) had $\mathrm{E}_{\mathrm{c}}, \varepsilon_{\mathrm{c}-\mathrm{y}}$, and $\sigma_{\mathrm{c}-\mathrm{y}}$ values similar to the gels with no plasticizers, perhaps due to the balance between plasticizing and anti-plasticizing behavior of glycerol at the $30 \%$ concentration.

\section{B. Effect of temperature and pressure on gel properties}

Chemical polymerization was utilized over the photochemical method for better control of gelation, especially for relatively thick gels. The chemically-crosslinked PEG-GLY (20\% PEGDA with 30\% GLY) gels fabricated in a heated die [Fig. 3(a)] showed an increase in opacity as temperature increased [Fig. 3(c)], consistent with the response of poly( $\mathrm{N}$-isopropylacrylamide) (PNIPA) in which lower fabrication temperatures produced translucent gels while those at higher 
temperatures turned opaque. ${ }^{30}$ Temperatures $>80{ }^{\circ} \mathrm{C}$ resulted in damaged gels. The measured compression properties generated noticeable trends. Batch variability was small [Fig. 3(d)-3(e)], suggesting uniformity and repeatability of the chemical gelation process. ANOVA tests revealed significant differences among temperature groups for all the mechanical properties except for the ultimate compressive stress $(\mathrm{p}=0.113)$. Gels fractured at $199 \pm 48 \mathrm{kPa}$ of compressive pressure. PEG-GLY gels made at $37^{\circ} \mathrm{C}$ had significantly higher yield stress (at $52 \pm 11 \mathrm{kPa}$ ) compared to the other temperature groups $(\mathrm{p} \leq 0.0308)$ [Fig. 3(d)]. It was observed that as temperature increased, the compressive strain values also increased [Fig. 3(e)]. Gels polymerized at $80{ }^{\circ} \mathrm{C}$ were the most flexible, with yield and ultimate strains of $19 \pm 2 \%$ and $70 \pm 2 \%$, respectively. Additionally, these gels $\left(\right.$ at $\left.80{ }^{\circ} \mathrm{C}\right)$ produced significantly $(\mathrm{p} \leq 0.0102)$ lower $\mathrm{E}_{\mathrm{c}}$ at $89 \pm 39 \mathrm{kPa}$ compared to the other temperature groups at $345 \pm 63 \mathrm{kPa}$. Heating increased the molecular motion and randomness of PEG chains' orientation, producing increased light absorbance (opacity) and spring ${ }^{31}$ behavior.

Application of constant gauge pressure of $90 \mathrm{MPa}$ using a pellet press [Fig. 3(b)] at room temperature produced PEG-GLY gels that had statistically-lower $E_{c}(p=0.0112)$ at $36 \pm 2 \mathrm{kPa}$ and $\sigma_{\mathrm{c}-\mathrm{y}}(\mathrm{p}=0.0228)$ at $5 \pm 2 \mathrm{kPa}$ compared to those made at ambient pressure. The incidence of untestable gels were high due to the die set leakiness at the pressurized condition; thus, the pellet press system needs improvement.

Pairwise comparison of PEG gels revealed that at $80{ }^{\circ} \mathrm{C}$, those with GLY had statistically lower ultimate compressive strength $(136 \pm 57 \mathrm{kPa}$ vs. $310 \pm 42 \mathrm{kPa} ; \mathrm{p}=0.0194)$ and compressive modulus ( $89 \pm 39 \mathrm{kPa}$ vs. $294 \pm 55 \mathrm{kPa} ; \mathrm{p}=0.0329)$ than without any plasticizer [Fig. 4(a)]. However, at $37^{\circ} \mathrm{C}$, the addition of GLY led to PEG gels with increased yield stress $(\mathrm{p}=0.031)$ and yield strain $(\mathrm{p}=0.0216)[$ Fig. 4(b)]. GLY also generated gels with higher $(\mathrm{p}=$ 0.0402) ultimate compressive strain at $21^{\circ} \mathrm{C}$ [Fig. 4(b)].

\section{Chemical versus photochemical gelation}


PEG hydrogels were crosslinked and polymerized using chemical (APD and TEMED) and photochemical reactions (I2959 and light at $254 \mathrm{~nm}$ ). It was determined through mechanical testing that the compressive modulus of PEG gels with GLY were significantly greater $(\mathrm{p}=$ 0.0387) when photopolymerized than chemically gelled [Fig. 5]. Moreover, photochemical PEGonly gels had statistically higher yield strain $(\mathrm{p}=0.0143)$ and stress $(\mathrm{p}=0.0296)$ compared to those made using chemical crosslinking [Fig. 5].

\section{Stability of glycerol in PEG hydrogels}

The subsequent experiments employed PEG gels with glycerol as the chosen plasticizer. Accordingly, glycerol's retention within the PEG matrix was evaluated. The glycerol standard curve followed a linear trendline [Fig. $\left.6(\mathrm{a}) ; \mathrm{r}^{2}=0.96629\right]$ with the equation: $\mathrm{C}=(\mathrm{A}-$ 0.8137)/1.6809, where $\mathrm{C}=$ GLY concentration and $\mathrm{A}=$ absorbance at $193 \mathrm{~nm}$. At $193 \mathrm{~nm}, \mathrm{GLY}$ generated the highest positive signal difference compared to water, PBS, PEGDA, TEMED, and APS (possible substances in the collected liquid). Based on this linear equation, the mass release fraction values were calculated from light absorbances. It was found that the cumulative release of glycerol out of the PEG-GLY gels [Fig. 6(b)] was time-dependent and behaved according to the saturation equation $\left(r^{2}=0.94571\right): R=t /\left(t_{h}+t\right)$, where $t_{h}$ was determined to be 0.9535 day $=$ time needed to release half the amount of GLY from the gel. At the 1-week experimental endpoint, PEG-GLY gels had diffused out $89 \pm 39 \%$ of the original glycerol mass load, demonstrating that glycerol was not covalently-bound to the PEG matrix and leached out quickly into the PBS medium. It is thus advised that PEG-GLY gels are used in minimal liquid environment to prevent glycerol release.

\section{E. Blood clotting on gels with bioactive glass particles}

Using kilns [Fig. 7(a)] for melting and annealing, bioglass particles were successfully synthesized [Fig. 7(b)], then incorporated into PEG-based gels. Blood coagulation on gel 
surfaces [Fig. 7(c)] revealed that PEG-GLY-BGHU1 gels (PEG-GLY hydrogels with BGHU1 bioactive glass particles on exposed surfaces) induced significantly faster clotting time per unit area compared to poly(styrene) surfaces (no gel; $\mathrm{p}=0.0207)$ and to PEG only $(\mathrm{p}=0.0334)$ gels

[Fig. 7(d)]. The blood coagulation on PEG-GLY-BGHU1 was also lower at $63 \pm 11 \mathrm{~s} / \mathrm{cm}^{2}$ versus on PEG-GLY at $81 \pm 21 \mathrm{~s} / \mathrm{cm}^{2}$, but statistically not significant $(\mathrm{p}=0.7721)$. Gels with bioglass (PEG-GLY-BG45S5 and PEG-GLY-BGHU1) activated similar rates of blood clotting ( $\mathrm{p}=$ 0.9998), implying that the new bioactive glass mixture (BGHU1) and the control Bioglass 45S5 were alike in their hemostatic capabilities. Other bioactive glass formulations had also been shown to increase hemostasis and in vitro blood coagulation. ${ }^{32,33}$ Faster blood clotting time may suggest better host tissue integration but ultimately requires actual in vivo implantation experiments.

\section{F. Biomechanical properties of gels with bioglass, alginate, and laponite additives}

PEG-GLY-BGHU1 gels had compressive modulus, yield strain, and yield strength results statistically-similar ( $p$ > 0.05) to gels (PEG-GLY) without the bioglass particles (Table II). Bioactive glass particles were only added on the gel surfaces, hence, did not alter the bulk properties. PEG-GLY hydrogels with added alginate (at 2.5\%) and laponite (at 2.5\%) constituents (PEG-GLY- BGHU1-ALG-LAP) produced higher $\mathrm{E}_{\mathrm{c}}(\mathrm{p} \leq 0.0431), \varepsilon_{\mathrm{c}-\mathrm{y}}(\mathrm{p} \leq 0.014)$, and $\sigma_{\mathrm{c}-\mathrm{y}}(\mathrm{p} \leq 0.0187)$ measurements compared to the groups without ALG and LAP (Table II). The ultimate strain and stress points of PEG-GLY-BGHU1 gels were determined to be $34 \pm 1 \%$ and $254 \pm 31 \mathrm{kPa}$, respectively. In another study using PEG gels, the presence of alginate and laponite additives also increased the mechanical strength of gels, particularly the fracture toughness or ultimate compressive strength due to the supplemental alginate network formation and reinforcing laponite load-bearing structures. ${ }^{21}$ The 79-kPa yield strength of the PEG-based gel IV disc model was below the $100 \mathrm{kPa}$ computed biomechanical tolerance of a native lumbar IV disc of an average man in supine position ${ }^{34}$, therefore the gel assembly needs further research 
and development.

PEG-GLY-BGHU1-ALG-LAP gels were geometrically modeled as cylindrical lumbar spine IV discs [Fig. 8(a)] and tested for cyclic compression [Fig. 8(b)] at an initial compressive strain of 4\% (determined to be within the linear elasticity region of the gel). Hysteresis and creep responses were observed during cycles of compression (loading) and relaxation (unloading) [Fig. $8(c)$ ], with similarities to behavior of actual IV discs during cyclic compression. ${ }^{35,36}$ Stress-strain curves were found to decrease in magnitude at increasing number of cycles (n) [Fig. 8(d)]. Consequently, hysteresis behavior and dissipated energy density $(\mathrm{E}=$ area within the hysteresis loop) also decreased following a power regression $\left(r^{2}=0.97\right)$ with the equation: $E=\alpha n^{\beta}$, where $\alpha$ $=4.5102 \mathrm{~J} / \mathrm{m}^{3}=$ dissipated energy density at the first cycle and $\beta=-0.4069=$ exponent $[$ Fig.

$8(e)]$, indicative of elastic recovery ${ }^{37}$ exhibited by biological tissue specimens. ${ }^{38,39}$

\section{CONCLUSIONS}

This research study showed improvements of compressive strengths of 20\% PEG gels through the use of GRAS plasticizers (sorbitol, glycerol, propylene glycol and PEG 8000) at relatively low saturation concentrations. Photochemical reaction proved to be the superior method of gelation over chemical for creation of stronger gels; but chemical means provided better control and product uniformity. The gelation temperature of $37^{\circ} \mathrm{C}$ made constructs (PEG with 30\% glycerol) with the highest yield strength among the other temperature groups. Gels synthesized at ambient pressure, with the addition of alginate and laponite, led to greater increase in the mechanical performance. These gels with surface bioglass particles, modeled to cylindrical lumbar intervertebral discs, demonstrated decent biomechanical responses. Additional experimentation and development activities are needed for translational applications.

\section{ACKNOWLEDGMENTS}


The authors would like to thank the members of the Bioengineering Materials Lab including

Daniel Foyt and Hazel Consunji for technical assistance, manuscript proofreading, and

discussion, Tyler Lavertu for gel fabrication under extreme pressure, Paul Chaleff and Bethany

Dill for help with bioglass synthesis, John Foy for providing the animal blood samples, Meir

Wieder for the Excel Visual Basic for Applications (VBA) coding, Pierre Llanos and Robert

Cerro for 3D printing assistance, and Jacqueline Scarola and Lori Castoria for help with reagents

purchasing. Support for this study was provided by Hofstra University internal research funds.

\section{REFERENCES}

1. M.S. Hahn, J.S. Miller and J.L. West: Three-Dimensional Biochemical and Biomechanical Patterning of Hydrogels for Guiding Cell Behavior Advanced Materials. 18(20), 2679 (2006).

2. P.N. Patel, C.K. Smith and C.W. Patrick: Rheological and recovery properties of poly (ethylene glycol) diacrylate hydrogels and human adipose tissue Journal of biomedical materials research Part A. 73(3), 313 (2005).

3. K. Arcaute, B.K. Mann and R.B. Wicker: Stereolithography of three-dimensional bioactive poly (ethylene glycol) constructs with encapsulated cells Annals of biomedical engineering. 34(9), 1429 (2006).

4. G.M. Cruise, D.S. Scharp and J.A. Hubbell: Characterization of permeability and network structure of interfacially photopolymerized poly (ethylene glycol) diacrylate hydrogels Biomaterials. 19(14), 1287 (1998).

5. S. Halstenberg, A. Panitch, S. Rizzi, H. Hall and J.A. Hubbell: Biologically engineered protein-graft-poly (ethylene glycol) hydrogels: a cell adhesive and plasmin-degradable biosynthetic material for tissue repair Biomacromolecules. 3(4), 710 (2002).

6. S. Varghese, D. van der Schaft and A. van Spreeuwel: Improving the properties of PEGDA hydrogels by adding clay particles, without reducing the biocompatibility Biomechanics and Tissue engineering. 9, 36 (2009).

7. J.C. Iatridis, S.B. Nicoll, A.J. Michalek, B.A. Walter and M.S. Gupta: Role of biomechanics on intervertebral disc degeneration and regenerative therapies: What needs repairing in the disc and what are promising biomaterials for its repair? The spine journal : official journal of the North American Spine Society. 13(3), 243 (2013).

8. E.M. Schutgens, M.A. Tryfonidou, T.H. Smit, F.C. Oner, A. Krouwels, K. Ito and L.B. Creemers: Biomaterials for intervertebral disc regeneration: past performance and possible future strategies Eur Cell Mater. 30, 210 (2015).

9. M. Khandaker, A. Orock, S. Tarantini, J. White and O. Yasar: Biomechanical performances of networked Polyethylene Glycol Diacrylate (PEGDA): Effect of photo initiator concentration, temperature, and incubation time.

10. F.T. Sejidov, Y. Mansoori and N. Goodarzi: Esterification reaction using solid heterogeneous acid catalysts under solvent-less condition Journal of Molecular Catalysis A: Chemical. 240(1), 186 (2005).

11. M.G.A. Vieira, M.A. da Silva, L.O. dos Santos and M.M. Beppu: Natural-based plasticizers and biopolymer films: A review European Polymer Journal. 47(3), 254 (2011).

12. M.L. López-Castejón, C. Bengoechea, M. García-Morales and I. Martínez: Effect of plasticizer and storage conditions on thermomechanical properties of albumen/tragacanth based bioplastics Food and Bioproducts Processing. 95, 264 (2015).

13. E. Vesterinen, T. Suortti and K. Autio: Effects of preparation temperature on gelation properties and molecular structure of high-amylose maize starch Cereal chemistry. 78(4), 442 (2001).

14. B. Kim: Effects of $\mathrm{pH} /$ temperature on the swelling behavior and rheological properties of hydrogel, City, 2010).

15. J.E. Elliott, M. Macdonald, J. Nie and C.N. Bowman: Structure and swelling of poly (acrylic acid) hydrogels: effect of $\mathrm{pH}$, ionic strength, and dilution on the crosslinked polymer structure Polymer. 45(5), 1503 (2004). 
16. D.S. Jones, G.P. Andrews and S.P. Gorman: Characterization of crosslinking effects on the physicochemical and drug diffusional properties of cationic hydrogels designed as bioactive urological biomaterials $J$ Pharm Pharmacol. 57(10), 1251 (2005).

17. N. Annabi, S.M. Mithieux, A.S. Weiss and F. Dehghani: The fabrication of elastin-based hydrogels using high pressure $\mathrm{CO}(2)$ Biomaterials. 30(1), 1 (2009).

18. J.L. Bron, L.A. Vonk, T.H. Smit and G.H. Koenderink: Engineering alginate for intervertebral disc repair Journal of the mechanical behavior of biomedical materials. 4(7), 1196 (2011).

19. S.N. Pawar and K.J. Edgar: Alginate derivatization: a review of chemistry, properties and applications Biomaterials. 33(11), 3279 (2012).

20. H. Tomas, C.S. Alves and J. Rodrigues: Laponite(R): A key nanoplatform for biomedical applications? Nanomedicine. (2017).

21. S. Hong, D. Sycks, H.F. Chan, S. Lin, G.P. Lopez, F. Guilak, K.W. Leong and X. Zhao: 3D printing of highly stretchable and tough hydrogels into complex, cellularized structures Adv Mater. 27(27), 4035 (2015). F. Baino, S. Hamzehlou and S. Kargozar: Bioactive Glasses: Where Are We and Where Are We Going? J Funct Biomater. 9(1), (2018).

23. R.C. de Guzman and S.Y. Rabbany: PEG-immobilized keratin for protein drug sequestration and $\mathrm{pH}-$ mediated delivery J Drug Deliv. 2016, 1 (2016).

24. B. Sheen and R.C. de Guzman: Electroresponsive PEG-chitosan matrix for anion release Biomaterials and Tissue Technology. 1(2), 1 (2017).

25. D.C. Greenspan and L.L. Hench: Chemical and mechanical behavior of bioglass-coated alumina Journal of biomedical materials research. 10(4), 503 (1976).

26. Y. Zhang, C. Rempel and Q. Liu: Thermoplastic starch processing and characteristics-a review Critical reviews in food science and nutrition. 54(10), 1353 (2014).

27. A. Lerbret, P.E. Mason, R.M. Venable, A. Cesaro, M.L. Saboungi, R.W. Pastor and J.W. Brady: Molecular dynamics studies of the conformation of sorbitol Carbohydr Res. 344(16), 2229 (2009).

28. P. Kaewprachu, K. Osako and S. Rawdkuen: Effects of plasticizers on the properties of fish myofibrillar protein film J Food Sci Technol. 55(8), 3046 (2018).

29. Y.R. Wagh, H.A. Pushpadass, F.M. Emerald and B.S. Nath: Preparation and characterization of milk protein films and their application for packaging of Cheddar cheese J Food Sci Technol. 51(12), 3767 (2014).

30. S. Dutta and D. Dhara: Effect of preparation temperature on salt-induced deswelling and pattern formation in poly (N-isopropylacrylamide) hydrogels Polymer. 76, 62 (2015).

31. K. Ciepluch, A. Radulescu, I. Hoffmann, A. Raba, J. Allgaier, D. Richter and R. Biehl: Influence of PEGylation on Domain Dynamics of Phosphoglycerate Kinase: PEG Acts Like Entropic Spring for the Protein Bioconjug Chem. 29(6), 1950 (2018).

32. T.A. Ostomel, Q. Shi and G.D. Stucky: Oxide hemostatic activity Journal of the American Chemical Society. 128(26), 8384 (2006).

33. S. Pourshahrestani, N.A. Kadri, E. Zeimaran, N. Gargiulo, S. Samuel, S.V. Naveen, K. Hasikin, T. Kamarul and M.R. Towler: Comparative efficacy of hemorrhage control of a novel mesoporous bioactive glass versus two commercial hemostats Biomed Mater. 13(2), 025020 (2018).

34. H.J. Wilke, P. Neef, M. Caimi, T. Hoogland and L.E. Claes: New in vivo measurements of pressures in the intervertebral disc in daily life Spine (Phila Pa 1976). 24(8), 755 (1999).

35. H. Schmidt, A. Shirazi-Adl, C. Schilling and M. Dreischarf: Preload substantially influences the intervertebral disc stiffness in loading-unloading cycles of compression J Biomech. 49(9), 1926 (2016).

36. S. Li, A.G. Patwardhan, F.M. Amirouche, R. Havey and K.P. Meade: Limitations of the standard linear solid model of intervertebral discs subject to prolonged loading and low-frequency vibration in axial compression $J$ Biomech. 28(7), 779 (1995).

37. L. Salari-Sharif, T.A. Schaedler and L. Valdevit: Energy dissipation mechanisms in hollow metallic microlattices Journal of Materials Research. 29(16), 1755 (2014).

38. Y. Zhu, G. Kang, C. Yu and L.H. Poh: Logarithmic rate based elasto-viscoplastic cyclic constitutive model for soft biological tissues Journal of the mechanical behavior of biomedical materials. 61, 397 (2016).

39. M. Perrini, A. Mauri, A.E. Ehret, N. Ochsenbein-Kolble, R. Zimmermann, M. Ehrbar and E. Mazza: Mechanical and microstructural investigation of the cyclic behavior of human amnion Journal of biomechanical engineering. 137(6), 061010 (2015). 
TABLE I. Plasticizers used in bulk PEG gels.

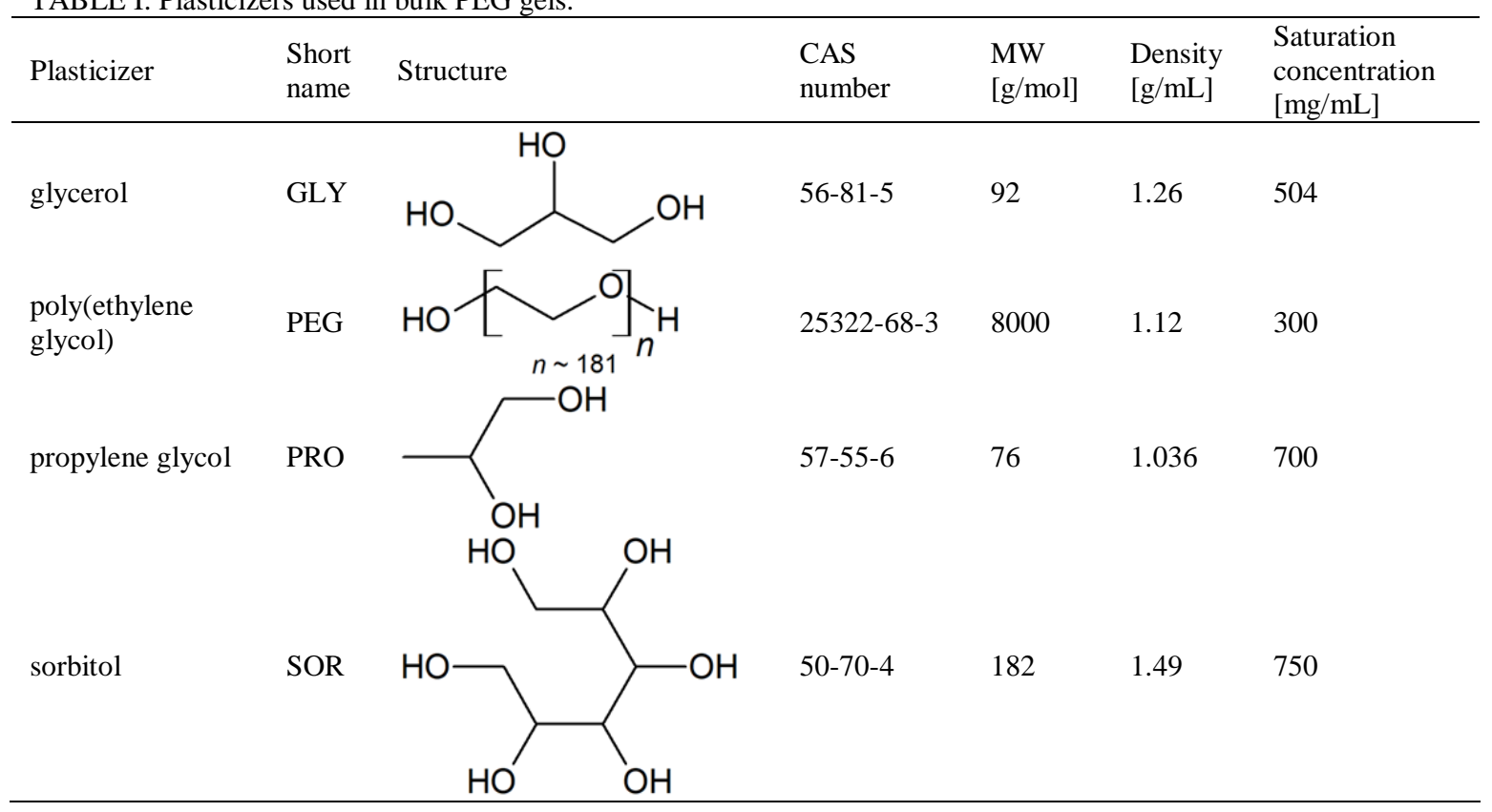

TABLE II. Compressive properties of PEG-GLY gels.

\begin{tabular}{llll}
\hline Gels & $\begin{array}{l}\text { Compressive } \\
\text { modulus }[\mathrm{kPa}]\end{array}$ & Yield Strain [\%] & Yield Stress [kPa] \\
\hline PEG-GLY & $383 \pm 64$ & $8 \pm 1$ & $25 \pm 7$ \\
PEG-GLY-BGHU1 & $346 \pm 103$ & $9 \pm 1$ & $30 \pm 12$ \\
PEG-GLY-BGHU1-ALG-LAP & $645 \pm 125^{*}$ & $12 \pm 1^{*}$ & $79 \pm 23^{*}$ \\
\hline
\end{tabular}

$* \mathrm{p}<0.05$. 
Fig. 1.
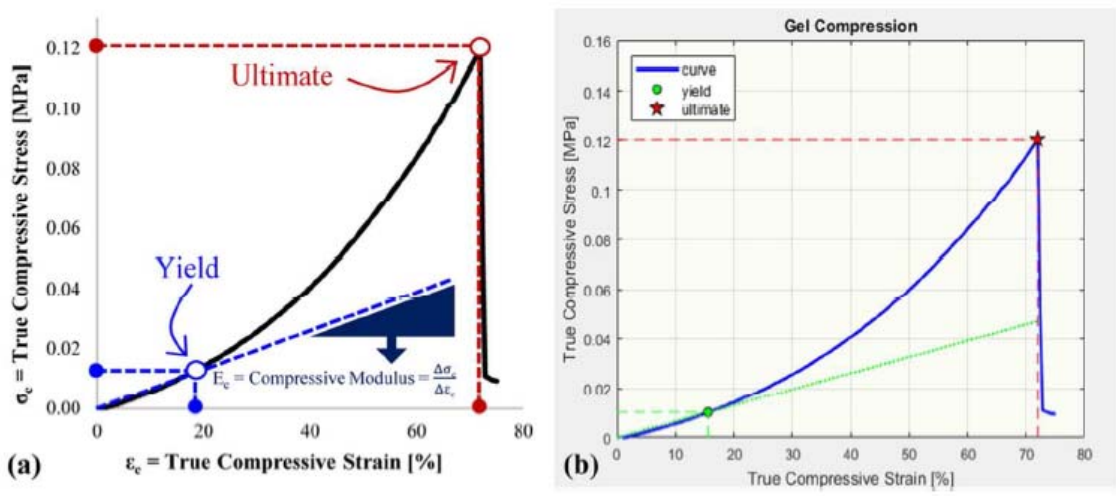

Representative stress-strain curves in (a) Excel and (b) MATLAB showing the yield and ultimate compressive stress and strain points. The slope of the linear elastic region is the compressive modulus.

Fig. 2.
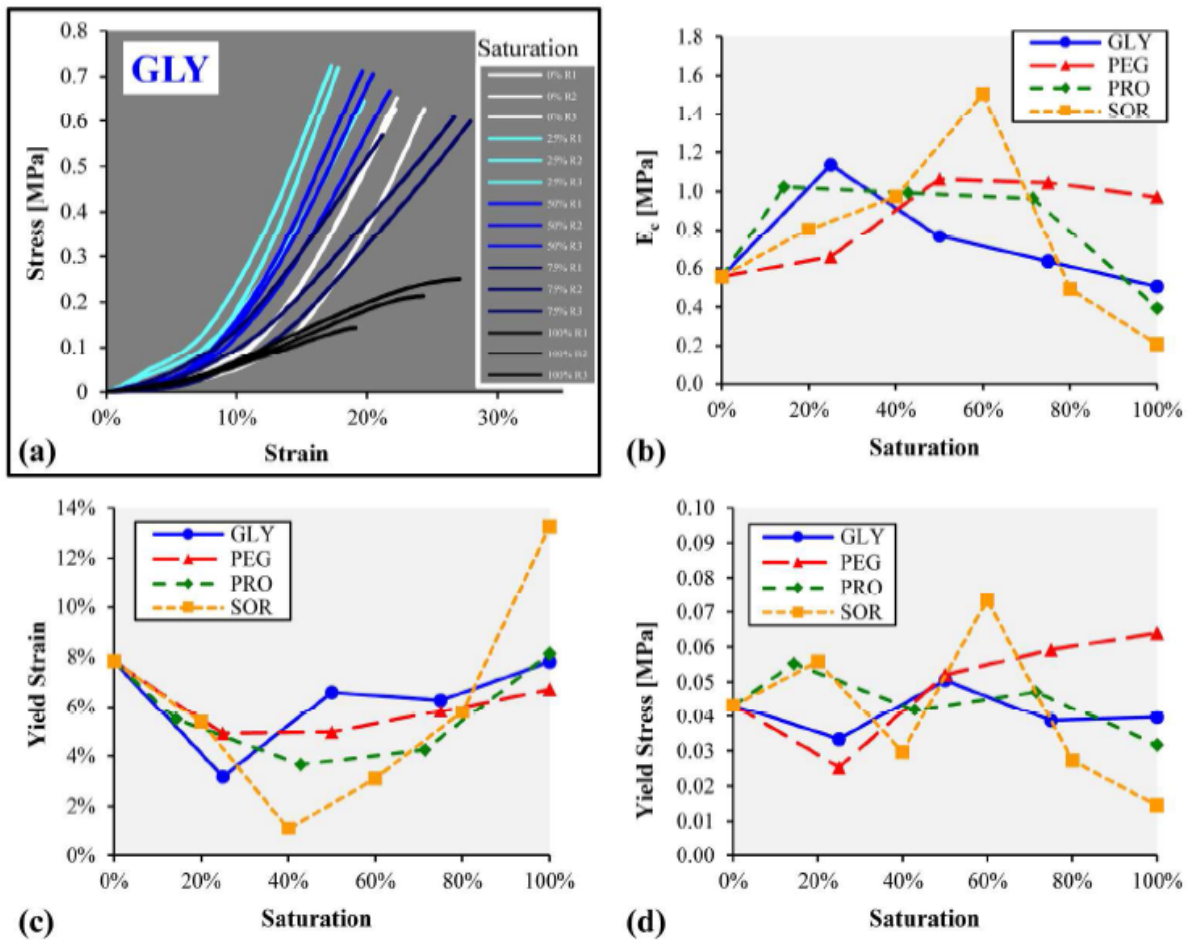

Compression properties of PEG gels with varying plasticizers (GLY, PEG, PRO, and SOR). (a) Stress-strain curves from triplicates (replicates: R1, R2, and R3) at increasing GLY. Normalized (b) compressive modulus, (c) yield strain, and (d) yield stress values fluctuate relative to the $0 \%$ plasticizer level (PEG gel only). 
Fig. 3.

(a)

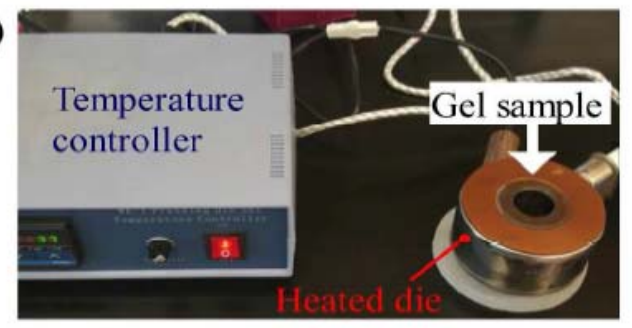

(e)

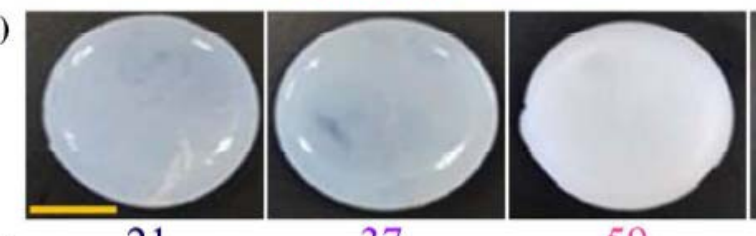

(b)
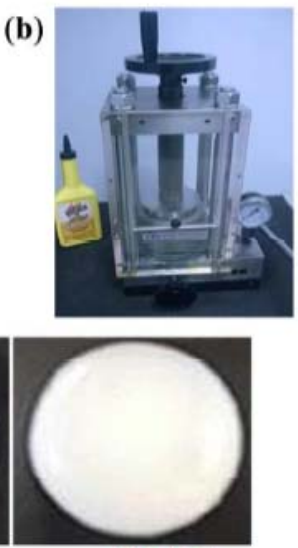

$80{ }^{\circ} \mathrm{C}$

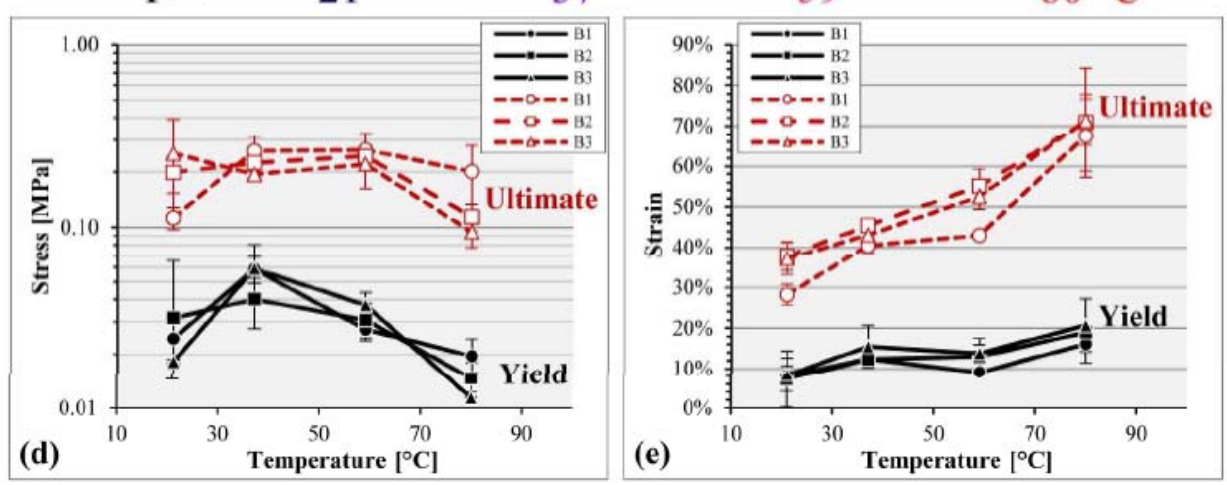

(a) The heated die set for temperature-controlled gelation. (b) Pellet press for application of constant pressure. (c) PEG-GLY gels (scale bar $=1 \mathrm{~cm}$ ) made at varying temperatures $(T)$ with yield and ultimate compressive (d) stresses and (e) strains from experimental batches (B1, B2, and B3), each in triplicates.

Fig. 4.
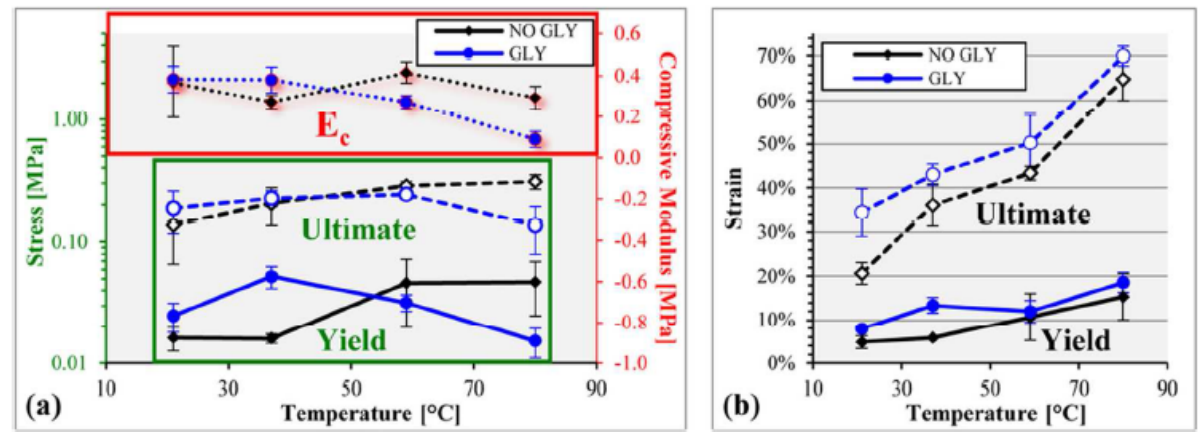

(a) Compressive stress and modulus, and (b) strain comparison between PEG gels without glycerol (NO GLY) and with glycerol (GLY) at various setting temperatures. 
Fig. 5.
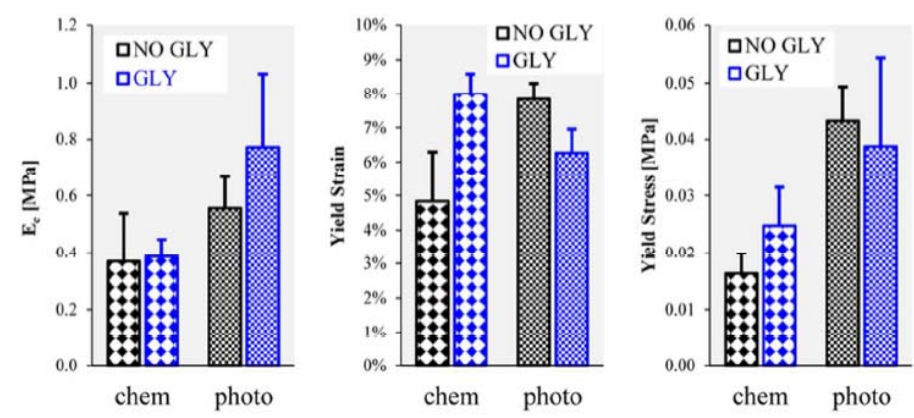

Properties of chemically (chem) and photochemically (photo) polymerized PEG gels.

Fig. 6.
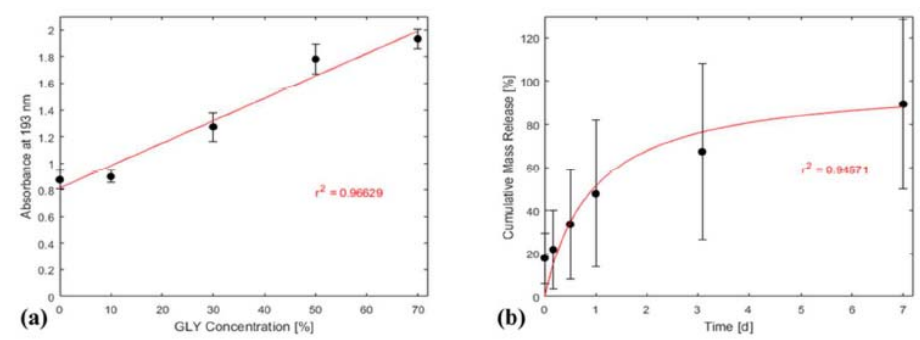

(a) Standard curve of absorbance versus glycerol concentration with a regression line. (b) Cumulative mass release of glycerol out of PEG-GLY gels over a one-week period with fitted saturation curve.

Fig. 7.
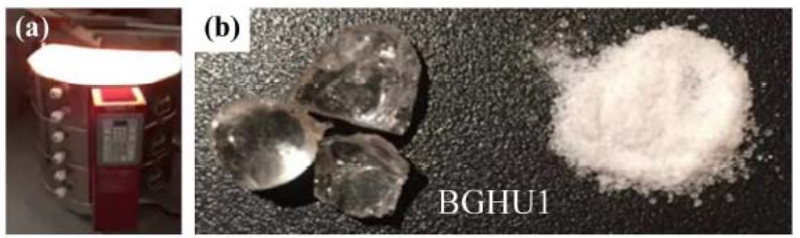

(c) blood on PG-BGHU1 gel
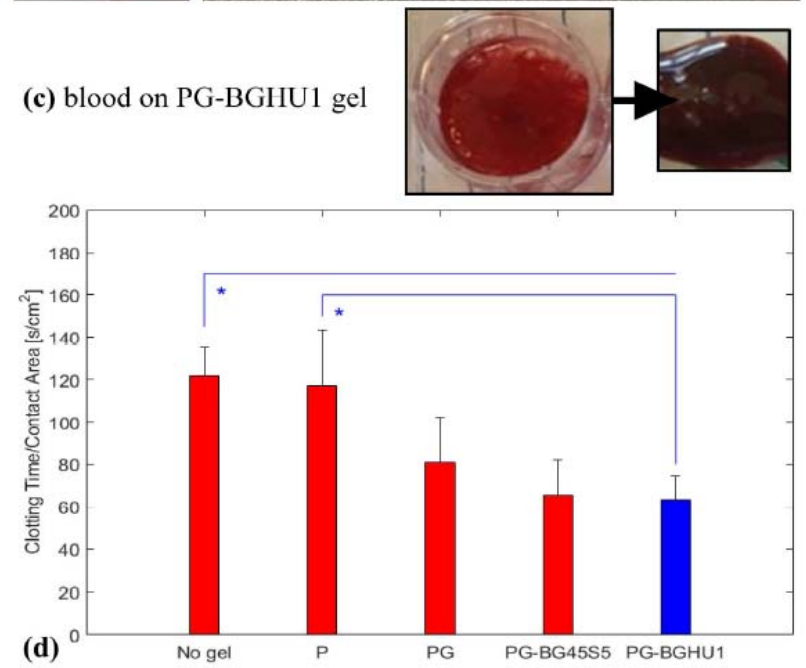

(a) Kiln for synthesis of (b) bioglass BGHU1. (c) A representative PEG-GLY-bioglass (PG-BGHU1) gel with blood, before and after coaqulation. (d) Clotting time per surface area versus substrate groups: No gel, PEG only (P), PEG-GLY (PG), and PEG-GLY with bioglass (PG-BG45S5 and PG-BGHU1) gels. * $\mathrm{p}<0.05$. 
bioRxiv preprint doi: https://doi.org/10.1101/405779; this version posted August 31, 2018. The copyright holder for this preprint (which was not certified by peer review) is the author/funder. All rights reserved. No reuse allowed without permission.

Fig. 8.

(a)
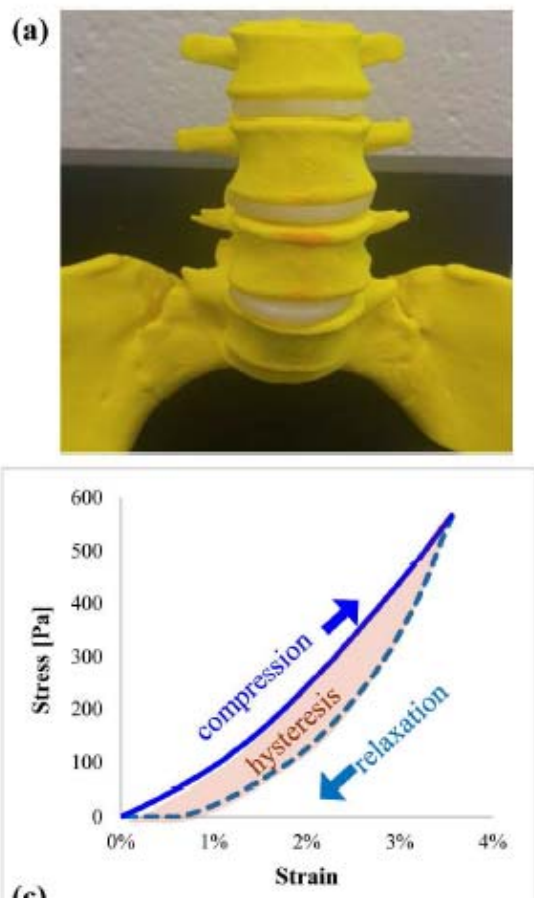

(c)

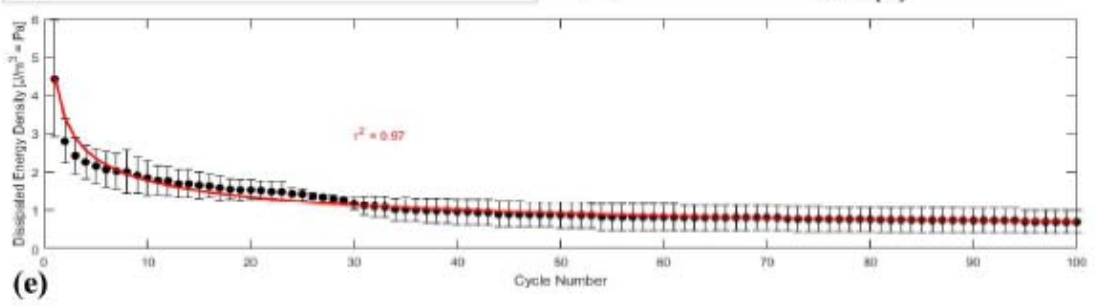

(b)
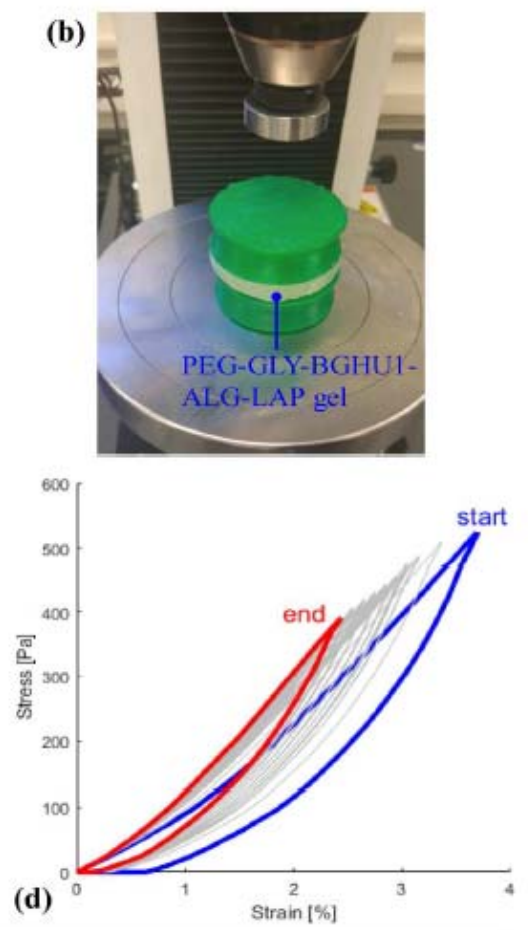

\section{(e)}

(a) Lumbar spine model with PEG-based gels as intervertebral discs. (b) Cyclic compression setup including the vertebral bodies that sandwich the experimental gel. (c) A representative cyclic loading (compression) and unloading (relaxation) stress-strain curve displaying the hysteresis loop. (d) 100 cycles of overlaid curves with the initial (start) and final responses (end). (e) Scatterplot of dissipated energy density versus cycle number with fitted power curve. 